\title{
Pseudotumour of the lung caused by infection with Bacillus sphaericus
}

\author{
P. ISAACSON, P. H. JACOBS, A. M. R. MACKENZIE, AND A. W. MATHEWS \\ From the Departments of Pathology and Microbiology, Southampton General Hospital \\ and the Wessex Cardiac and Thoracic Centre, Southampton
}

SYNOPSIS A patient is described who suffered from severe chronic asthma complicated by repeated chest infections. She developed a large gelatinous pseudotumour of the lung which was found to be caused by Bacillus sphaericus.

Human infections with organisms of the Bacillus genus other than anthrax are uncommon although, especially under conditions of decreased resistance, the pathogenicity of these organisms is well recognized (Farrar, 1963; Pearson, 1970; Ihde and Armstrong, 1973). The most commonly reported pathogen of this genus has been Bacillus subtilis but other members of the genus have been incriminated from time to time.

The following case report is the fourth in which Bacillus sphaericus has been documented as a pathogen, and it is unique in that the organism produced a massive pseudotumour of the lung.

\section{Case report}

The patient, who was born in 1939 , developed severe bronchial asthma at the age of 2 years after an attack of whooping cough. During childhood she also suffered from eczema, hay fever, and angioneurotic oedema. She was first seen in Southampton in 1963 when, at 24 years of age, she demonstrated a persistent grade II dyspnoea with central cyanosis and evidence of pulmonary hypertension with right ventricular hypertrophy. Chest $x$-rays showed widespread fine mottling throughout the lungs, and pulmonary function tests showed severe fixed airways obstruction. Skin tests were positive to a wide variety of antigens including Aspergillus fumigatus, but aspergillus precipitins were negative. The Mantoux test was negative. Treatment was started with prednisone, $5 \mathrm{mg}$ three times per day, and prophylactic isoniazid. Steroid therapy in one form or another was continued throughout the course of the illness. Over the next few years there was no significant change in the chest $x$-ray but there was some improvement of clinical status and pulmonary

Received for publication 10 February 1976 function. In 1965 she was able to visit a relative in 은 California, but she continued to suffer from attacks of severe wheezing dyspnoea associated with purulent $\mathcal{W}$ sputum. These episodes of infection became more frequent and severe. Klebsiella pneumoniae was isolated from the sputum and the patient was given frequent courses of ampicillin, gentamicin, and cotrimoxazole. In 1971 the chest $x$-ray showed an $\overrightarrow{0}$ increase in the diffuse lung shadowing, and there was oे a persistent leucocytosis of over $20 \times 10^{9} / 1$.

In November 1973, after her return from a second visit to California, she deteriorated considerably, and the chest $x$-ray showed a large area of consolidation in the anterior segment of the left upper lobe, which $\stackrel{\triangleright}{\mathbb{D}}$ gradually increased in size over the next year (fig 1). Bronchoscopy on two occasions showed the left upper lobe segmental bronchi to be occluded by thick mucus which could not be aspirated easily. Culture from bronchoscopy grew coliforms and Pseudomonas pyocyanea. Serum immunoglobulins were measured at this time and were normal. Skin tests and serology for coccidioidomycoses and histoplasmosis were negative.

In October 1974 she was admitted in terminal respiratory failure, and a soft fluctuant swelling was 웅 noted in the second left intercostal space anteriorly $>$ with a similar swelling over the sternum. She died shortly after admission, aged 35 years.

\section{NECROPSY}

The body was that of an emaciated young woman. A $\omega$ $6 \mathrm{~cm}$ soft subcutaneous mass was present in the left second intercostal space anteriorly, and a similare $3 \mathrm{~cm}$ mass bulged parasternally in the third inter- $\mathbb{D}$ costal space on the left. On reflecting the sternum it $\stackrel{?}{-}$ was apparent that these subcutaneous masses were $\frac{0}{0}$ extensions of a large, soft, mucoid tumour that $\frac{\vec{D}}{\mathbb{D}}$ 806 


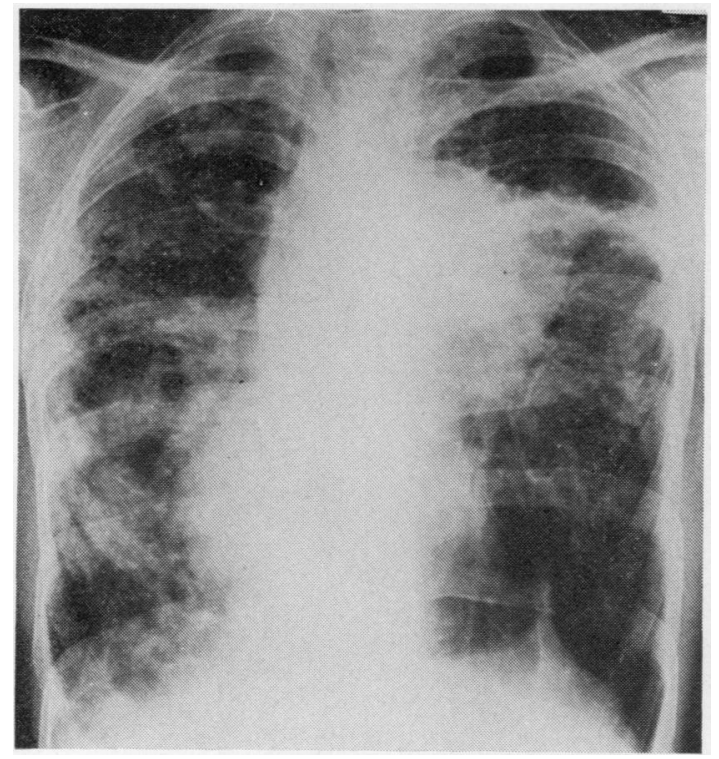

Fig 1 Chest $\mathrm{x}$-ray taken in November 1973 showing an area of consolidation in the left upper lobe.

replaced the medial half of the upper lobe of the left lung (fig 2). The tumour infiltrated the intercostal muscles and the mediastinum, where it closely surrounded the large vessels. The remainder of the left lung, and the right lung, showed marked fibrosis and basal bronchiectasis with scattered locules of pus and clear fluid. Fragments of the tumour were taken for bacteriological examination, as were swabs from the purulent foci.

Other significant macroscopic findings were obliterative fibrous pericarditis, right ventricular hypertrophy (the right ventricle weighing $76 \mathrm{~g}$ with a total heart weight of $290 \mathrm{~g}$ ), and atrophy of the adrenal glands, which together weighed $5 \mathrm{~g}$.

Microscopic examination of multiple sections of the mass in the left chest showed it to consist of masses of eosinophilic organisms. These were elongated, frequently curled, and coiled, and ranged in length from $3.75 \mu$ to $12.5 \mu$ and in width from $1.25 \mu$ to $2.5 \mu$. They were separated by fine fibrous bands and mixed with an infiltrate of neutrophil polymorphs (figs 3 and 4). A broad, fibrous band separated this process from the lung parenchyma but clumps of similar organisms were seen in bronchi of the left upper lobe. Gram stain showed that the organisms consisted of a Gram-negative sheath enclosing long bacilliform rods which were both Gram-positive and Gram-negative (fig 5). This Gram-negative material around the organisms stained faintly positive with periodic acid-Schiff but

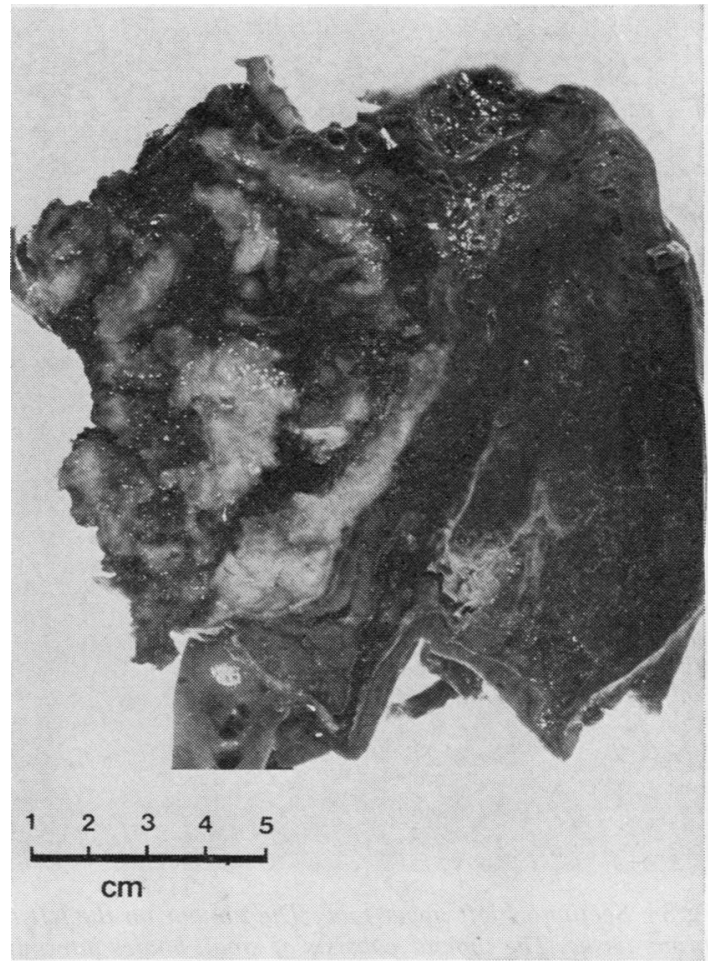

Fig 2 Sagittal section of left lung showing replacement of most of the left upper lobe by gelatinous tumour which encircles the great vessels.

mucicarmine, Ziehl Nielsen, and Grocott stains were all negative.

\section{BACTERIOLOGY}

A direct smear of the 'tumour' revealed a Gram variable bacillus similar to that seen in histological sections. No capsule was demonstrable but the organisms were surrounded by a viscous matrix.

Fungal and mycobacterial cultures were negative. No growth was obtained on horse blood agar after overnight incubation, but after four days' aerobic incubation at $37^{\circ} \mathrm{C}$ slight growth was obtained in glucose heart infusion broth. Gram stain revealed a pleomorphic Gram variable bacillus, $0.5 \mu$ to over $50 \mu$ in length (fig 6). Morphology was essentially similar to that seen in the tissue sections. Some unusual features included swelling and bulging of the cell wall with pseudobranching and serpiginous forms. 'Horseshoe' and 'boomerang' shapes were frequently seen in cultures incubated at $37^{\circ} \mathrm{C}$ but infrequently at $30^{\circ} \mathrm{C}$. Colonies were best established on horse blood agar and on heated chocolate agar and were an opaque, smooth, greyish-yellow with an 


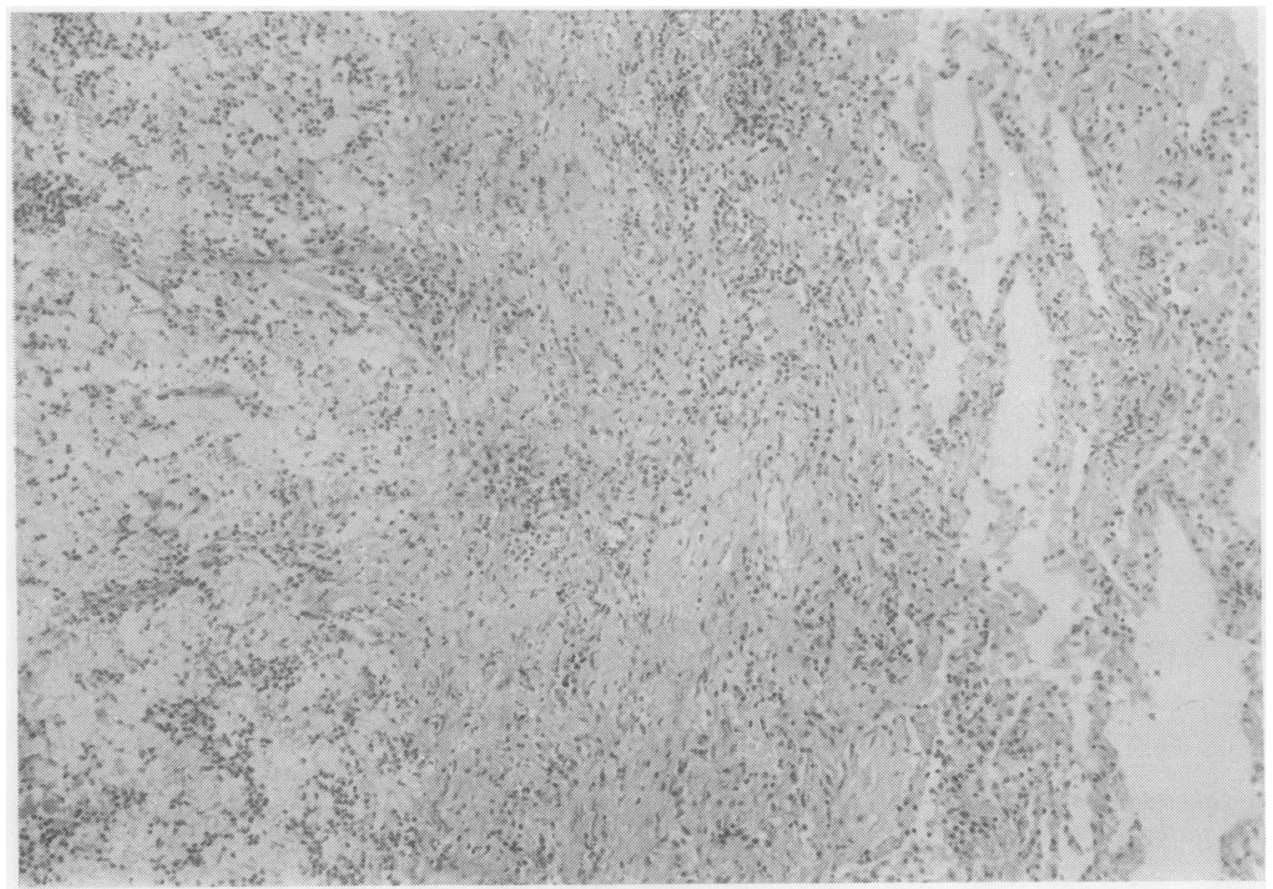

Fig 3 Section of left upper lobe. The tumour on the left is separated from the lung by a broad band of fibrous tissue. The tumour consists of small bodies intermingled with neutrophils and delicate fibrous tissue septa. (Haematoxylin and eosin $\times 83$ )

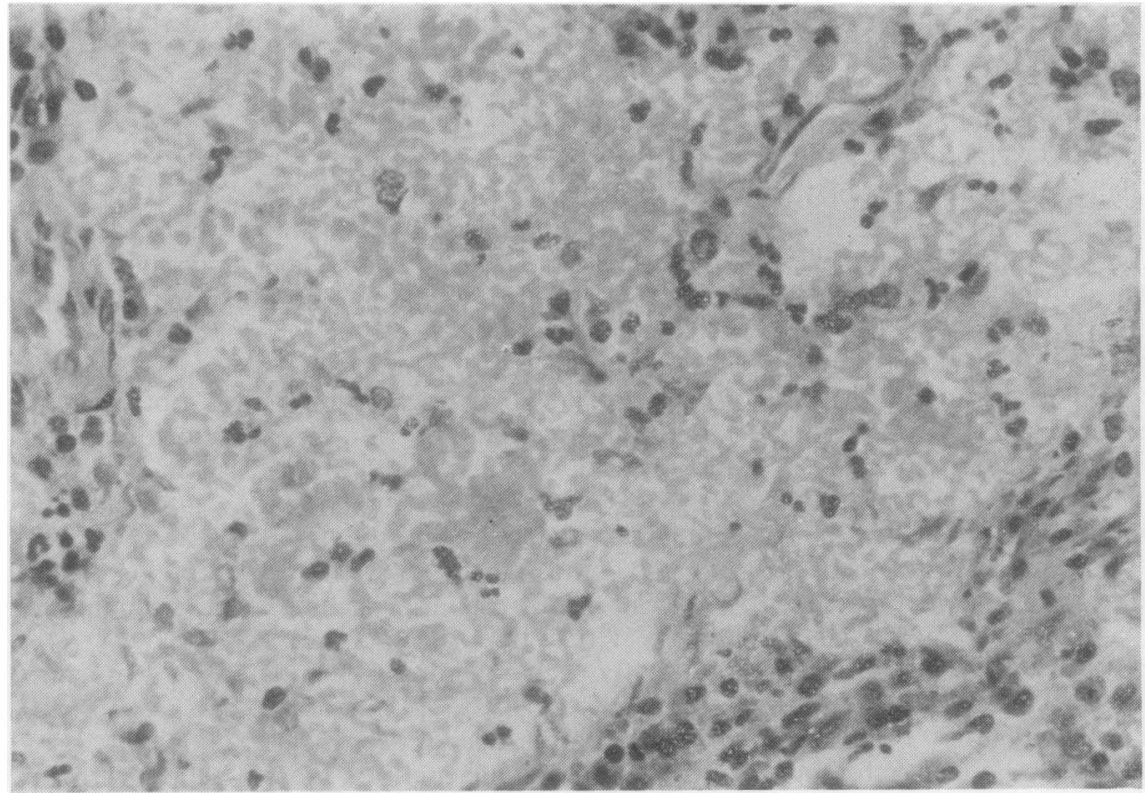

Fig 4 High-power detail of tumour showing masses of eosino-

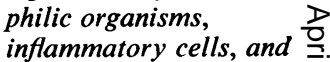
some fibrous tissue. (Haematoxylin and eosin $\times 300)$ 


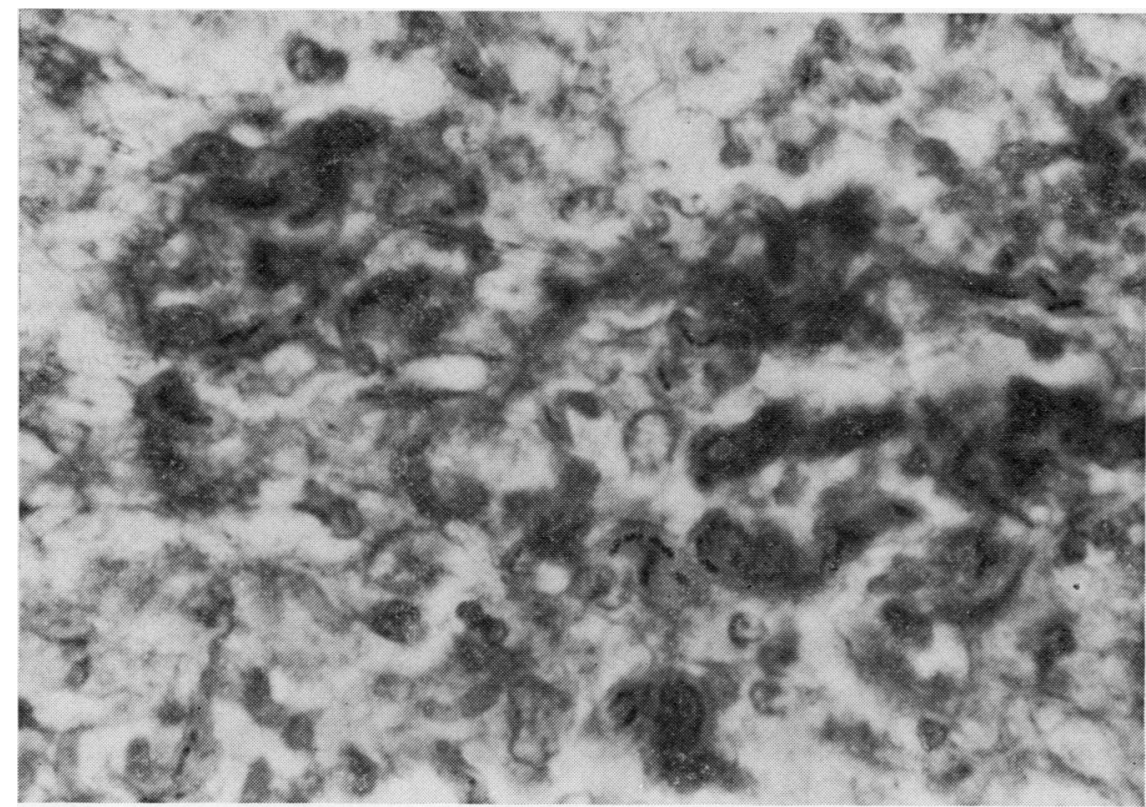

Fig 5 Gram stain of histological section of tumour. Gram variable pleomorphic bacilli are enclosed in a Gramnegative sheath. $(\times 750)$

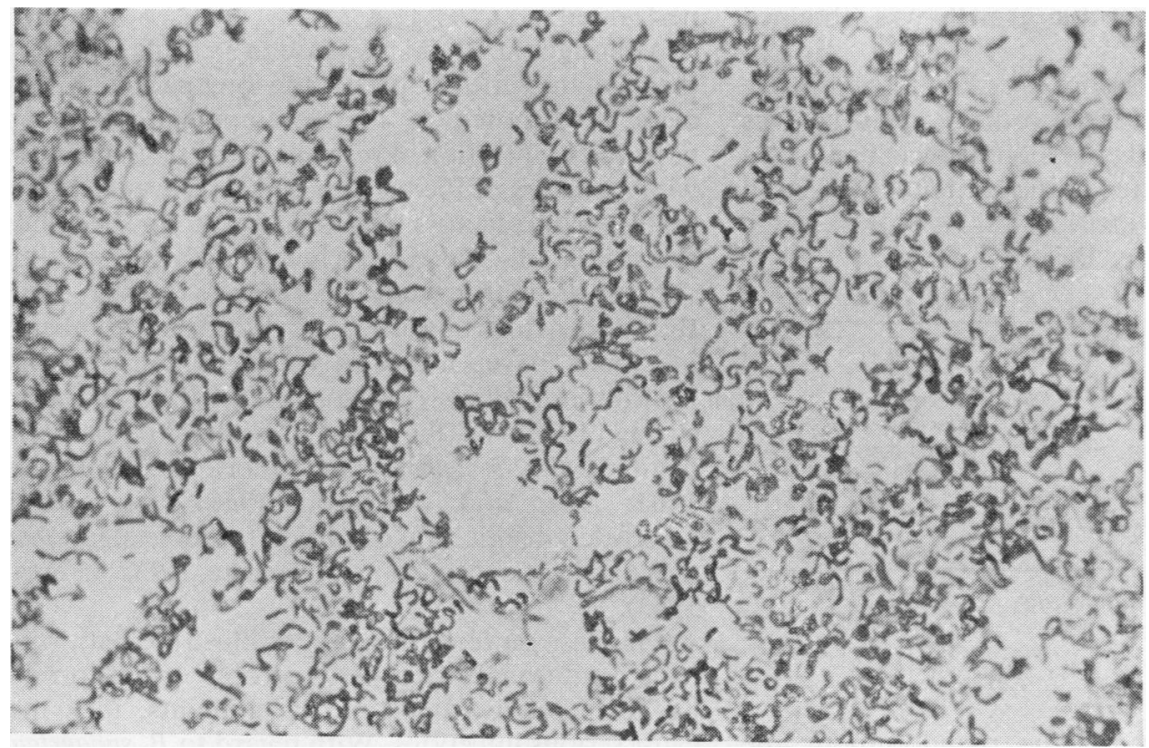

Fig 6 Gram stain of organism grown at $37^{\circ} \mathrm{C}$ showing Gram variability with marked pleomorphism.

$(\times 975)$

entire edge and a papillate centre having an average diameter of $1 \mathrm{~mm}$.

At $30^{\circ} \mathrm{C}$ growth was more easily established in both broth and plate cultures. Soft, emulsifiable colonies were up to $2 \mathrm{~mm}$ in diameter, flat and irregular, and exhibited a chrome yellow pigment with faint haemolysis. In contrast to organisms grown at $37^{\circ} \mathrm{C}$ (fig 6), incubation at the lower temperature produced a straight, more uniform rod (fig 7).

Motility, which lessened with increasing incubation temperature, was of a rapid gyrating type. After 


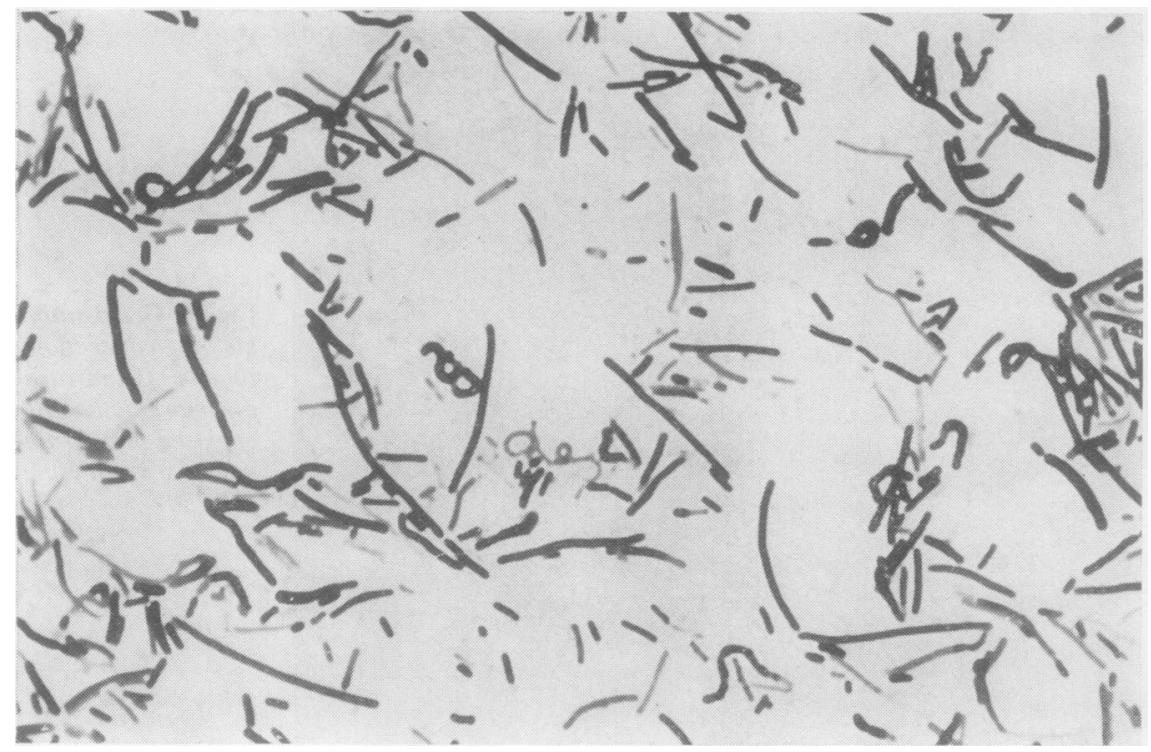

Fig 7 Gram stain of organism grown at. $30^{\circ} \mathrm{C}$ showing straight uniform Gram variabs rods. $(\times 975)$

one week spores were produced, most of which were spherical, subterminal or terminal with bulging of the sporangia. No anaerobic growth was obtained at $37^{\circ} \mathrm{C}$ or at $30^{\circ} \mathrm{C}$.

Biochemical characteristics are summarized in the table. The organism was accordingly identified as Bacillus sphaericus, and this identity was confirmed by Dr. L. R. Hill, Curator of the National Collection of Type Cultures, and Professor J. R. Norris, of the Meat Research Institute, Bristol.

\begin{tabular}{llllr}
\hline Oxidase - & Glucose - & Glycerol & - \\
Catalase - & Lactose - & Gluconate & - \\
VP & Sucrose - & Glycogen & - \\
Nitrate - & Mannitol - & Casein hydrolysis - \\
Indole - & Maltose - & Starch hydrolysis - \\
Urea - & Salicin - & Lecithin hydrolysis - \\
Citrate - & Dextrin - & \\
ONPG - & Fructose - & \\
$\mathrm{H}_{2} \mathrm{~S}-$ & Tylose - & \\
\hline
\end{tabular}

Table Biochemical characteristics

\section{Discussion}

Before the appearance of the pseudotumour the patient had developed persistent infection with a variety of pathogenic organisms. There is a well recognized group of patients with chronic asthma in whom persistent infection is a feature, and this is known to affect the prognosis unfavourably (Ogilvie, 1962). Although there was no evidence of immunoglobulin deficiency or fibrocystic disease in this patient it is possible that a defect of cellular immunity could have been responsible for repeated infections. No studies of lymphocyte function were, howevg, performed.

Although the initial response to steroids was go there can be little doubt that the long period of treatment predisposed to the development of an op- $\frac{\bar{O}}{\mathrm{O}}$ portunistic infection. Previous reports of infections with members of the bacillus genus have emphasized $\mathbb{Q}$ predisposing factors leading to diminished host $\vec{\Rightarrow}$ resistance which have included diabetes mellitus, $\frac{0}{3}$ chronic alcoholism, and malignancy (Farrar, 1963; Ihde and Armstrong, 1973). A significant number of infections have, however, occurred in previously healthy individuals (Pearson, 1970; Ihde and Arm-: strong, 1973). Of the three previously reported in-윽 fections with B. sphaericus (Lacorte, 1932; Farrar, 1963; Allen and Wilkinson, 1969), two had ano associated debilitating condition (chronic alcoholism and nephrotic syndrome) (Lacorte, 1932; Farrar,으 1963).

This case serves once again to illustrate that, whileㅡㅡㄹ it is traditional to regard aerobic spore-forming bacilli, when isolated, as contaminants, they may cause serious infections. With regard to B. sphaericus,, in particular, the ease of growth at $30^{\circ} \mathrm{C}$ as compared to $37^{\circ} \mathrm{C}$ has not been commented on previously. It is of interest, too, that in vitro morphology at $37^{\circ} \mathrm{C}$ soo closely resembled that seen in tissue sections.

The formation of a large pseudotumour in $a \stackrel{\infty}{?}$ patient has not to our knowledge previously been described in association with bacillus infections or, indeed, with any bacterial infection. The production 
of a viscous matrix by the organism, which accounted for much of the tumour bulk, has been noted in laboratory cultures of the bacillus group (Gibson, 1944) but not in human infections, although Ihde and Armstrong (1973) describe a gelatinous exudate from a necrotic axillary tumour infected with $B$. subtilis; the tumour, however, is not further characterized. Pulmonary cryptococcosis may result in the formation of well circumscribed fibrous tumours containing gelatinous foci (toruloma) (Spencer, 1968), and with the history of this patient's visit to California, it was this diagnosis that was suspected and led to bacteriological examination of what, at first sight, appeared to be an unusual mucusproducing adenocarcinoma of the lung.

We are grateful to Dr. L. R. Hill, of the National Collection of Type Cultures, to Professor J. R. Norris, Meat Research Institute, Langford, Bristol, for confirming the identity of the organism as $B$. sphaericus, and to Dr. W. Macleod, of the Western Hospital, Southampton, whose patient this was.

\section{References}

Allen, B. T. and Wilkinson, H. A. III (1969). A case of meningitis and generalized Schwartzman reaction caused by Bacillus sphaericus. Johns Hopk. med. J., 125, 8-13.

Farrar, W. E. Jr. (1963). Serous infections due to 'nonpathogenic' organisms of the genus Bacillus. Amer. J. Med., 34, 134-141.

Gibson, T. (1944). A study of Bacillus subtilis and related organisms. J. Dairy Res., 13, 248-260.

Ihde, D. C. and Armstrong, D. (1973). Clinical spectrum of infection due to Bacillus species. Amer. J. Med., 55, 839-845.

Lacorte, J. G. (1932). Bacillus serositis; new species (isolated from a human case of a primary inflammation of the serous membranes). Mem. Inst. Osw. Cruz, 26, 8-13.

Ogilvie, A. G. (1962). Asthma: a study in prognosis of 1000 patients. Thorax, 17, 183-189.

Pearson, H. E. (1970). Human infections caused by organisms of the Bacillus species. Amer. J. clin. Path., 53, 506-515.

Spencer, H. (1968). Pathology of the Lung, 2nd edition, pp. 300-301. Pergamon, Oxford. 